According to the authors, the success of valve repair in patients with deteriorating cardiac function is largely determined by the timing of intervention and the precision of surgery.

Claire Braybrook

Original article Yamaguchi $\mathrm{H}$ et al. (2006) Mitral valve repair in patients with infective endocarditis. Circ J70: 179-183

\section{Is aspirin effectiveness in cardiovascular disease prevention influenced by a patient's sex?}

The impact of a patient's sex on the effectiveness of aspirin treatment in the primary prevention of cardiovascular events in at-risk individuals is not clear. Berger et al. did a metaanalysis to investigate whether the advantages and disadvantages of aspirin treatment varied between sexes.

Six prospective, randomized, controlled trials (involving 95,456 individuals; 51,342 women) reporting the incidence of myocardial infarction (MI), stroke and cardiovascular mortality in patients assigned aspirin treatment or control treatment were included in the analysis. Compared with controls, aspirin therapy reduced the number of cardiovascular events by $12 \%$ in women and by $14 \%$ in men $(P=0.03$ and $P=0.01$, respectively). In women, aspirin reduced the risk of stroke by $17 \%(P=0.02)$, which was attributable to a reduced incidence of ischemic, but not hemorrhagic, stroke. Aspirin did not alter the risk of $\mathrm{Ml}$ in women. In men, aspirin did not reduce the risk of stroke but reduced the risk of $\mathrm{Ml}$ by $32 \%$ compared with controls $(P=0.001)$. Aspirin increased the risk of bleeding in men and women by similar amounts, but did not alter cardiovascular mortality in either group.

In summary, overall cardiovascular event rates are reduced by aspirin in both sexes, but via different routes. The differences in the response to aspirin were based on small numbers of Mls and strokes and need to be investigated in larger trials before clear conclusions can be drawn.

\section{Kate Matthews}

Original article Berger IS et al. (2006) Aspirin for the primary prevention of cardiovascular events in women and men: a sex-specific meta-analysis of randomized controlled trials. JAMA 295: 306-313

\section{Magnetic navigation system aids guidewire placement in $\mathrm{PCI}$}

Difficulty in navigating guidewires through complex coronary artery lesions can lead to failure of percutaneous coronary intervention (PCl). A magnetic navigation system, recently approved by the FDA and now tested in a clinical trial, could help circumvent these problems. The Niobe ${ }^{\circledR}$ system (Stereotaxis, Inc., St Louis, $\mathrm{MO}$ ) incorporates two permanent magnets that generate a $0.08 \mathrm{~T}$ magnetic field over the heart, a magnetic-tipped guidewire, and a software program to control the position of the magnets and deflect the guidewire. Navigation is viewed via a computer monitor.

A set of patients with tortuous or severely angulated coronary arteries underwent magnetassisted $\mathrm{PCl}$. For 46 patients this was the first attempt at intervention (primary group), whereas in 13 patients this was a re-intervention after conventional angioplasty had already failed (secondary group; mostly involving lesions within the circumflex artery territory). Crossing of the target lesion was largely successful, with $49(89 \%)$ of 55 lesions crossed in the primary group and $9(69 \%)$ of 13 in the secondary group. $\mathrm{PCl}$ was successful in $84 \%$ of primary cases and $62 \%$ of secondary cases. Although median times for crossing the lesion were longer in the secondary group than in the primary group-as were median procedure and fluoroscopy times - median time spent on guidewire placement in secondary procedures was less than in the initial, manual placement attempts.

Atmakuri et al. acknowledge that the equipment is in the early stages of development, but anticipate even greater success as the technology progresses.

Pippa Murdie

Original article Atmakuri SR et al. (2006) Initial experience with a magnetic navigation system for percutaneous coronary intervention in complex coronary artery lesions. JAm Coll Cardiol 47: 515-521

\section{A meta-analysis of self- management of oral anticoagulation}

Maintaining the INTERNATIONAL NORMALIZED RATIO (INR) within the therapeutic range and ensuring the safety of oral anticoagulant use requires frequent and accurate monitoring. To

\section{GLOSSARY} INTERNATIONAL NORMALIZED RATIO (INR)

A method developed by the WHO to standardize the prothrombin time, allowing direct comparison of prothrombin time values across laboratories 